\title{
Use of methyl methacrylate resin for embedding bone marrow trephine biopsy specimens
}

\author{
D Blythe, N M Hand, P Jackson, S L Barrans, R D Bradbury, A S Jack
}

\begin{abstract}
Aims-To evaluate the use of methyl methacrylate resin as an embedding medium for undecalcified bone marrow trephine biopsy specimens.

Methods-About 2500 undecalcified bone marrow trephine biopsy specimens were processed, and embedded in methyl methacrylate resin. Semithin sections $(2-3 \mu \mathrm{m})$ were stained by routine tinctorial and immunocytochemical staining methods with a wide range of antibodies using a standard streptavidin biotin horseradish peroxidase technique. Different antigen retrieval pretreatments were evaluated.

Results-Bone marrow trephine biopsy specimens are embedded routinely in methyl methacrylate at the Haematological Malignancy Diagnostic Service at The Leeds General Infirmary. Over 50 different primary antibodies are in current use; for the majority of these, microwave antigen retrieval or trypsin digestion, or both, is either essential or greatly enhances the results.
\end{abstract}

Conclusions-Embedding bone marrow trephine biopsy specimens in methyl methacrylate resin retains morphology and permits reliable, high quality immunocytochemistry. This is particularly desirable for the demonstration of neoplastic cells in regenerative marrow after chemotherapy, and in the detection of residual disease after treatment. The use of methyl methacrylate for routine use on bone marrow trephine biopsy specimens is advocated.

(F Clin Pathol 1997;50:45-49)

Keywords: methyl methacrylate resin; bone marrow trephine biopsy; morphology; immunocytochemistry.

The investigation of neoplastic and other disorders of the bone marrow requires morphological examination of a cellular aspirate and a trephine biopsy specimen in conjunction with immunophenotypic and molecular biological techniques. Two- or three-colour flow cytometry is currently the method of choice for the immunophenotypic characterisation of cell populations in bone marrow aspirate samples. However, immunohistological studies on bone marrow trephine biopsy specimens also have an important and complementary role. The trephine biopsy specimen is particularly important in cases where the aspirate sample is inadequate for flow cytometric investigation or where a population of tumour cells is poorly represented in the aspirate. This is most common where marrow infiltration is focal, or where fibrosis is associated with the tumour.

Decalcified paraffin wax embedded biopsy specimens can be studied using immunohistochemistry, but this technique has the disadvantage that morphology and antigenicity may be poorly preserved. Resin section histology offers the prospect of excellent high resolution light microscopy, and both acrylic and epoxy resins have been advocated as being suitable for this purpose. ${ }^{1-4}$ The most popular resin is that based on the monomer glycol methacrylate (GMA). Various GMA mixtures have been used and most of these will give high quality morphological preservation, but the results of immunohistological studies have been disappointing. Although there are reports that immunohistochemistry is possible on GMA embedded tissue, ${ }^{5-8}$ we agree with others that the techniques are complicated, troublesome and impractical in the routine laboratory. ${ }^{9}$ For this reason, decalcification and paraffin wax embedding continues to be widely used in the immunohistological investigation of bone marrow trephine biopsy specimens. ${ }^{10-13}$

In an attempt to overcome the problems associated with the GMA resin, a method based on a methyl methacrylate (MMA) resin has been reported ${ }^{14}{ }^{15}$ which retains good morphological detail and permits the use of standard immunohistological methods. This paper describes the application of this method to routine diagnostic practice, based on the experience of processing around 2500 bone marrow trephine biopsy specimens.

\section{Methods}

SPECIMEN PROCESSING

Specimens were fixed for a minimum of 18 hours in $10 \%$ formalin. Other formalin based fixatives such as formol saline, neutral buffered formalin and formol calcium have also been evaluated and produce similar results. Bone marrow trephine biopsy specimens were not decalcified prior to processing. The tissue was processed manually in small glass vials on a roller mixer (automated tissue processors may be used for the dehydration stages). The tissue was dehydrated through $50 \%, 70 \%$ and $95 \%$ ethanol for a minimum of one hour in each, and $100 \%$ ethanol for two hours minimum.

After dehydration, the tissue was incubated overnight at room temperature in the resin mixture, consisting of a 3:1 solution of methyl methacrylate and dibutyl phthalate, on a roller mixer in a fume cupboard. The tissue was then

embedded in heavy duty polyethylene
Correspondence to:

Accepted for publication 25 October 1996 


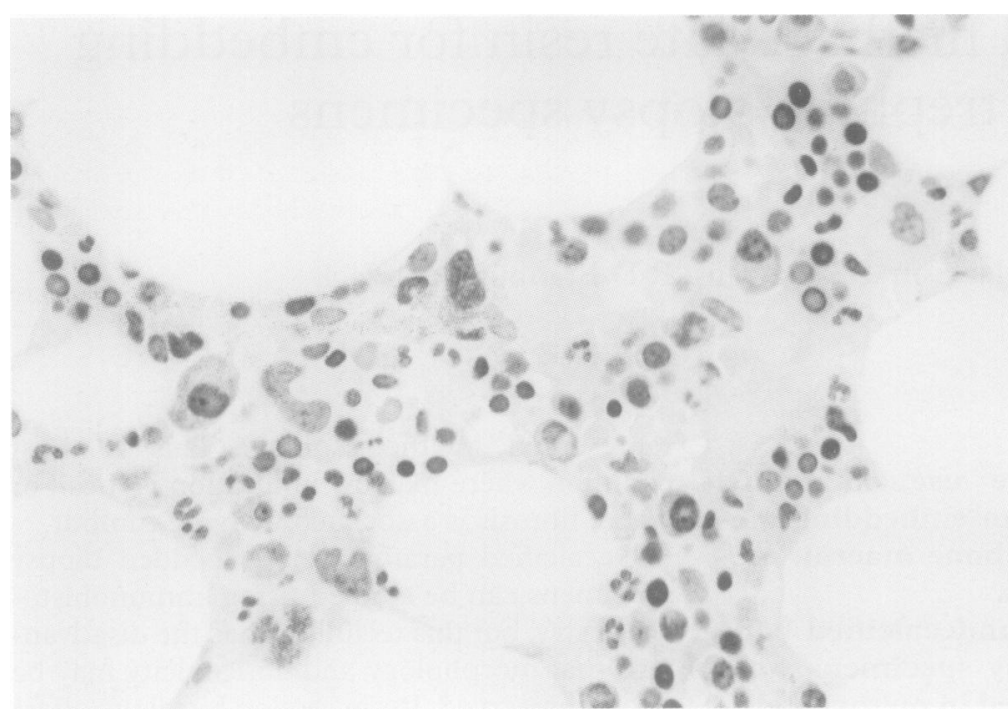

Figure 1 Early infiltration by myeloma (haematoxylin and eosin). This specimen shows low level interstitial infiltrate of plasma cells. The improved morphology associated with the use of $M M A$ is clearly evident.

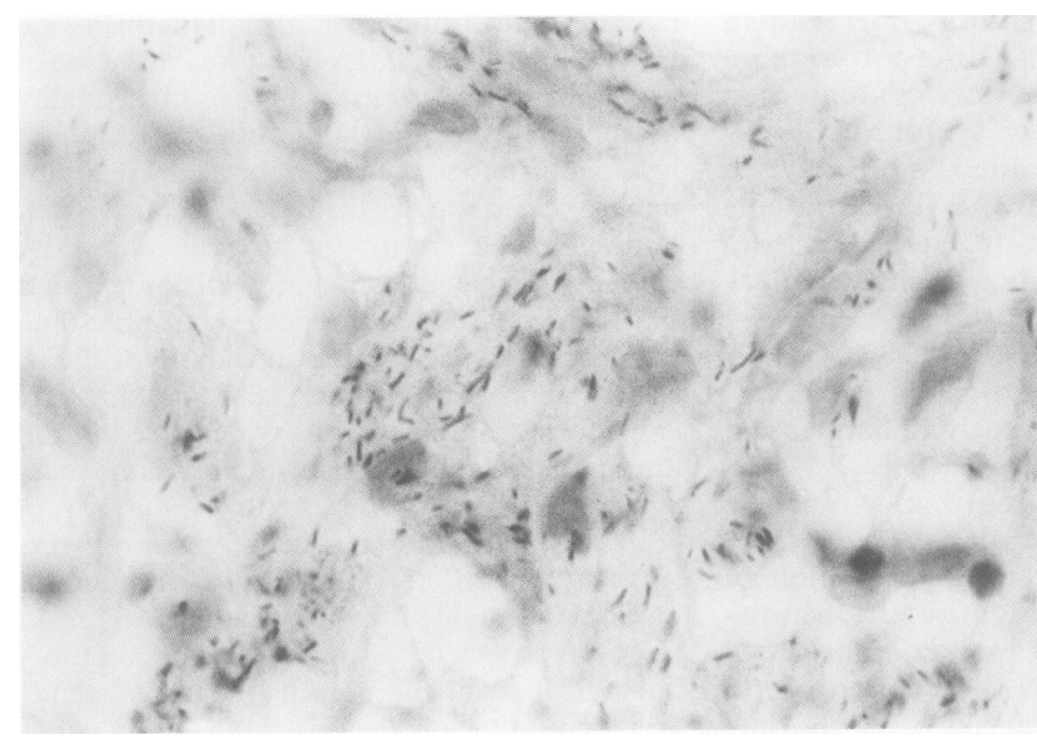

Figure 2 HIV infection (Ziehl-Neelsen). This HIV positive patient developed pancytopenia. The bone marrow biopsy specimen showed a granulomatous infiltrate containing very large numbers of mycobacteria. moulding cup trays with a block stub, and an identification label attached. The embedding formulation consisted of a fresh 3:1 methyl methacrylate/dibutyl phthalate mixture with $5 \%$ pre-dried benzoyl peroxide and $1 \% \mathrm{~N}, \mathrm{~N}$ dimethylalinine. Benzoyl peroxide is potentially explosive in the dry state and is supplied dampened; aliquots are dried overnight at $37^{\circ} \mathrm{C}$. MMA acts as the monomer, dibutyl phthalate as the plastisicing/softening agent, benzoyl peroxide as the catalyst, and $\mathrm{N}, \mathrm{N}$ dimethylaniline as the accelerator. The embedding mixture was allowed to polymerise for a minimum of two hours at room temperature in a desiccator until the resin solidified. Because oxygen inhibits polymerisation of acrylic resins, including MMA, some laboratories prefer to polymerise in an anaerobic environment, either by using a vacuum or by displacing the air with oxygen-free nitrogen. We have found that these methods are not necessary, as the resin stubs seal the resin mixture into the mould sufficiently to prevent oxygen contamination. All infiltration and embedding procedures should be done in a fume cupboard.

Following polymerisation, the sections were cut at $2 \mu \mathrm{m}$ using glass Ralph knives on a Reichert-Jung 1140 Autocut. Sections were floated out on a $56^{\circ} \mathrm{C}$ distilled water bath, picked up on clean slides, drained, and placed on a hot plate for a minimum of 10 minutes. Sections for reticulin demonstration were cut at $4 \mu \mathrm{m}$, and those for immunocytochemistry at $3 \mu \mathrm{m}$. These were picked up on slides coated with $2 \%$ aminopropyltriethoxysilane in acetone (APES), and either placed on a hot plate for 20 minutes, or dried overnight at $60^{\circ} \mathrm{C}$.

TINCTORIAL STAINING

Prior to staining, MMA resin was removed with xylene at $37^{\circ} \mathrm{C}$ for 20 minutes. Sections were then rinsed in alcohol and washed in water prior to conventional histological staining. Routinely, the following tinctorial stains are applied to all bone marrow trephine biopsy specimens: haematoxylin and eosin (fig 1) May-Grunwald Giemsa (MGG); Gordon and Sweet's reticulin ammoniacal silver stain; and Perls Prussian blue. A wide range of tinctorial staining techniques may be applied to MMA sections (fig 2 ).

IMMUNOHISTOLOGICAL METHODS

The MMA resin was removed from the sections as described earlier. Endogenous peroxidase activity was blocked using $0.5 \%$ hydrogen peroxide in methanol for 30 minutes. The optimal antigen retrieval pretreatment procedures will depend upon the antibodies under investigation, and are shown in tables $1-4$.

Microwave antigen retrieval

Sections were placed in $400 \mathrm{ml} 0.01 \mathrm{M}$ citrate buffer ( $\mathrm{pH}$ 6.0) (using plastic staining racks and a microwave resistant plastic container). The sections were then microwaved (Panasonic NN6402) at high power $(800 \mathrm{~W})$ for eight minutes, allowed to cool for five minutes, and irradiated again for a further three minutes.
Figure 3 Post-treatment multiple myeloma (VS38c). This specimen was taken as part of routine follow up. The use of VS38c, which in the haemopoietic system is a specific marker of plasma cells, shows an interstitial infiltrate of plasma cells corresponding with a low level of residual disease. 
Table 1 Optimal antigen demonstration after microwave antigen retrieval

\begin{tabular}{llll}
\hline Antibody/clone & Species & Source & Dilution \\
\hline IgA & Polyclonal & Dako & 1 in 800 \\
IgG & Polyclonal & Dako & 1 in 400 \\
Kappa & Polyclonal & Dako & 1 in 400 \\
Lambda & Polyclonal & Dako & 1 in 300 \\
CD15 (Leu M1) & Monoclonal & Becton Dickinson & 1 in 10 \\
CD20 (L26) & Monoclonal & Dako & 1 in 100 \\
CD34 (QBend 10) & Monoclonal & Oxoid & 1 in 100 \\
CD43 (L20) & Monoclonal & Dako & 1 in 50 \\
CD45 (PD7/26+2B11) & Monoclonal & Dako & 1 in 20 \\
CD45RO (UCHL1) & Monoclonal & Dako & 1 in 100 \\
MB2 & Monoclonal & Europath & 1 in 20 \\
CD68 (PG-M1) & Monoclonal & Dako & 1 in 100 \\
CD68 (KP1) & Monoclonal & Dako & 1 in 100 \\
BLA.36 (A27-42) & Monoclonal & Dako & 1 in 50 \\
DBA.44 & Monoclonal & Dako & 1 in 10 \\
Bcl-2 (124) & Monoclonal & Dako & 1 in 50 \\
BNH-9 & Monoclonal & Dako & 1 in 25 \\
EMA (E29) & Monoclonal & Dako & 1 in 200 \\
HLA-DR (TAL.1B5) & Monoclonal & Dako & 1 in 500 \\
LMP-1 (CS 1-4) & Monoclonal & Dako & 1 in 10 \\
Glycophorin C (Ret 40f) & Monoclonal & Dako & 1 in 100 \\
OPD4 & Monoclonal & Biogenex & 1 in 50 \\
PGP 9.5 & Polyclonal & Ultraclone & 1 in 1000 \\
P53 (pAB 1801) & Monoclonal & HMDS & 1 in 10 \\
S100 & Polyclonal & Dako & 1 in 200 \\
Tdt & Polyclonal & Harland Seralab & 1 in 20 \\
Vimentin (V9) & Monoclonal & Dako & 1 in 50 \\
Desmin (DE-R-11) & Monoclonal & Europath & 1 in 10 \\
Plasma cell (VS38c) & Monoclonal & Dako & 1 in 100 \\
J-Chain & Polyclonal & Biogenex & Neat \\
\hline Source Becton Dickinson & Ltd Betwen Tws & Cowey & \\
\hline
\end{tabular}

Source: Becton Dickinson UK Ltd, Between Towns Road, Cowley OX4 3LY, UK; Biogenex, distributed by Biomen Ltd, Pentos House, Falcon Business Park, Ivanhoe Road, Finchampstead, Berks RG11 4QQ; Dako Ltd, 16 Manor Courtyard, Hughenden Avenue, High Wycombe, Bucks HP13 5RE; Europath Ltd, Highland Comfort, Union Hill, Stratton, Bude, Cornwall EX23 9BL; Harland Seralab Ltd, Hophurst Lane, Crawley Down, Sussex RH10 4FF; HMDS Laboratory, Algernon Firth Building, Institute of Pathology, The General Infirmary at Leeds, Leeds LS1 3EX; Immunotech, distributed by Coulter Electronics Ltd, Northwell Drive, Luton, Beds LU3 3RH; Novocastra Laboratories Ltd, 24 Claremont Place, Newcastle upon Tyne NE2 4AA; Ultraclone Ltd, Rossiters Farmhouse, Wellows, Isle of Wight PO41 0TE.

Table 2 Optimal antigen demonstration using trypsinisation followed by microwave antigen retrieval

\begin{tabular}{llll}
\hline Antibody/clone & Species & Source & Dilution \\
\hline IgD & Polyclonal & Dako & 1 in 50 \\
IgM & Polyclonal & Dako & 1 in 50 \\
CD1a (010) & Monoclonal & Immunotech & Neat \\
CD3 & Polyclonal & Dako & 1 in 100 \\
CD8 (C8/144B) & Monoclonal & Dako & 1 in 50 \\
CD21 (IF8) & Monoclonal & Dako & 1 in 20 \\
CD23 (1B12) & Monoclonal & Novocastra & 1 in 10 \\
CD30 (Ber H2) & Monoclonal & Dako & 1 in 15 \\
CD45RA (MT2) & Monoclonal & Biogenex & 1 in 10 \\
CDw75 (LN-1) & Monoclonal & Novocastra & 1 in 20 \\
CD79a (JCB117) & Monoclonal & Dako & 1 in 25 \\
Ki-67 (MIB-1) & Monoclonal & Immunotech & 1 in 50 \\
\hline
\end{tabular}

Table 3 Optimal antigen demonstration after trypsin or microwave antigen retrieval

\begin{tabular}{llll}
\hline Antibody/clone & Species & Source & Dilution \\
\hline CAM 5.2 & Monoclonal & Becton Dickinson & 1 in 2 \\
Factor VIII & Polyclonal & Dako & 1 in 500 \\
Poly Keratin & Polyclonal & Dako & 1 in 200 \\
\hline
\end{tabular}

Table 4 Optimal antigen demonstration with no antigen retrieval

\begin{tabular}{llll}
\hline Antibody/clone & Species & Source & Dilution \\
\hline CD57 (HNK-1) & Monoclonal & HMDS & 1 in 20 \\
Myeloperoxidase & Polyclonal & Dako & 1 in 4000 \\
\hline
\end{tabular}

After leaving to cool for 20 minutes, the sections were washed in cold water.

Trypsinisation antigen retrieval

Sections were incubated in $0.025 \%$ trypsin $(100 \mathrm{mg}$ trypsin, $400 \mathrm{ml}$ distilled water, $8 \mathrm{ml}$ $5 \%$ calcium chloride, $\mathrm{pH}$ to 7.8 with $1 \%$ sodium hydroxide) at $37^{\circ} \mathrm{C}$ for six minutes, and then washed in cold tap water to prevent further digestion.

\section{Trypsinisation and microwave antigen retrieval} Trypsinisation was done prior to microwave irradiation, using the methods described earlier.

Following antigen retrieval, sections were rinsed in $0.005 \mathrm{M}$ Tris buffered saline (TBS) (pH 7.6), and incubated in $20 \%$ normal serum for 10 minutes. Excess serum was drained from the sections, and primary antibody applied for an incubation period of one hour, using $0.01 \%$ Tween 20 in TBS as the antibody diluent. Sections were then washed in TBS for five minutes, and incubated in biotinylated secondary antibody (Dako Duet kit K492) for 30 minutes. After washing in TBS, sections were incubated in streptavidin/biotin/horseradish peroxidase complex (Dako Duet kit K492) for 30 minutes. Following washing in TBS, sections were developed in $0.02 \%$ diaminobenzidene in Tris buffer ( $\mathrm{pH} 7.6)$ containing $0.15 \%$ hydrogen peroxide. Sections were developed for 10 minutes, then washed in water, and intensified in $0.5 \%$ copper sulphate in $0.9 \%$ sodium chloride for five minutes. After washing in water, the sections were counterstained in haematoxylin. Sections can be immunostained either manually or by semi-/ automatic methods. Throughout the staining procedures, it is important to recognise that etched MMA sections dry out quicker than paraffin wax sections when not covered with fluid, and, consequently, this must be avoided to help prevent background staining.

\section{Results}

The method described here is routinly used at the Haematological Malignancy Diagnostic Service at Leeds General Infirmary. Approximately 2500 bone marrow biopsy specimens have been processed in this way. In every case haematoxylin and eosin, Giemsa, reticulin, and Perls stained sections are examined. Sections are studied immunohistologically when necessary, and about 3000 resin sections are examined using immunocytochemistry each year. None of the biopsy specimens embedded in MMA in our department have been difficult to interpret because of technical failure.

Forty six antibodies are currently in routine use. With the exception of myeloperoxidase and CD57, microwave antigen retrieval or trypsin digestion, or both, are either essential or greatly improve staining intensity and reproducibility. ${ }^{16}$ The optimal antigen retrieval pretreatment methods and antibody dilutions are shown in tables $1-4$.

Figures 3-6 show representative MMA sections stained immunocytochemically with a number of routinely used antibodies.

\section{Discussion}

Based on our experience described here, we conclude that MMA embedding is the method of choice for processing bone marrow trephine biopsy specimens. The technique has several advantages over other previously reported resin embedding methods. The most important of 


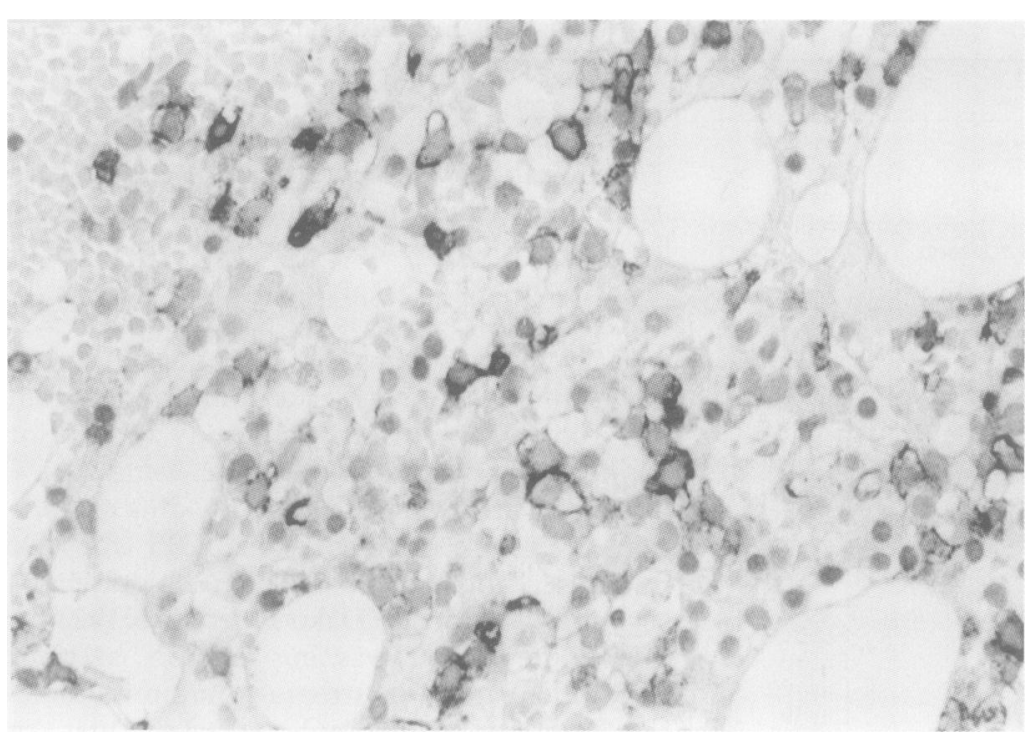

Figure 4 Myelodysplastic syndrome (CD34). This patient presented with pancytopenia. Examination of the bone marrow showed abnormal architecture and trilineage dysplasia. The number of blast cells was shown using anti-CD34.

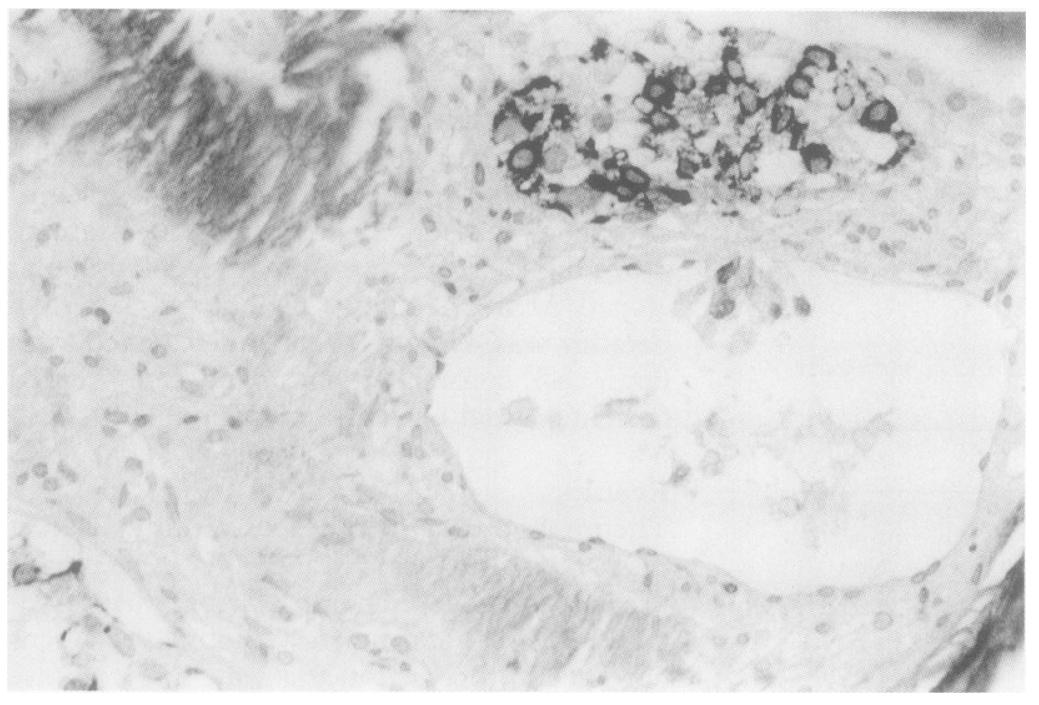

Figure 5 Metastatic carcinoma (cytokeratin). This patient presented with leuco-lerythroblastic changes in the peripheral blood. The bone marrow was replaced by dense fibrosis. Using an antibody directed against cytokeratin (Cam 5.2), a number of small clusters of metastatic carcinoma were identified.

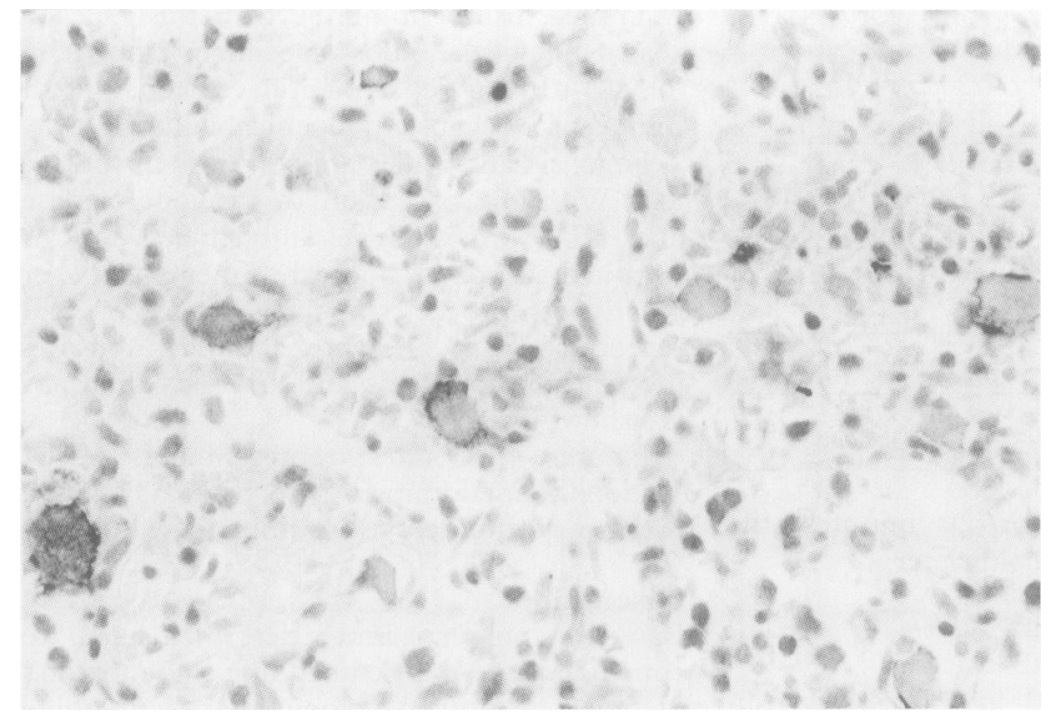

Figure 6 Post-transplantation lymphoproliferative disorder (LMP-1). This patient presented with pancytopenia several years after a kidney transplant. The marrow showed a nodular infiltrate which included large atypical cells, some of which resembled Reed-Sternberg cells. These cells expressed the Epstein-Barr virus protein LMP-1. these is that the resin is soluble in its polymerised state in xylene, and unlike other resins MMA does not undergo superpolymerisation. ${ }^{17}$ Therefore, sections can be cut and immunostained long after the biopsy specimen was originally embedded. After removal of the resin, the section can be hydrated and handled as a paraffin wax section using optimal antigen retrieval pretreatments as required. Almost all of the antibodies that can be used on paraffin wax sections also work well on MMA sections (CD40 being an exception). However, pretreatment schedules may need to be prolonged for MMA sections. Our processing method is done entirely at room temperature after conventional histological fixation in $10 \%$ formalin. The standard of morphological preservation is consistently better than that on decalcified paraffin wax sections (fig 1). Variables which may be encountered with paraffin wax sections, such as the length of time in decalcification fluid and the microtomy of thin sections, are not a problem with MMA sections. A further important advantage of this method is that routinely stained sections are available for examination within 48 hours of the specimen being taken. This compares favourably with the use of decalcification followed by paraffin wax processing and embedding.

The diagnostic application in which this technique has been of most value is the identification of small populations of neoplastic cells, particularly in regenerative marrow following chemotherapy. Suspicious cells are more readily identified in semi-thin MMA sections, and these can then be identified positively using appropriate markers. This can be of value in detecting residual disease after treatment of myeloma, acute lymphoblastic leukaemia, or hairy cell leukaemia. Low levels of marrow infiltration by follicle centre lymphoma can also be detected. In all of these applications, it is essential to correlate closely the cytological appearance of the cell with the antibody reactivity. This is much easier to do on MMA sections than on decalcified paraffin wax sections.

Another important area of application is in the assessment of the marrow in cases of suspected myelodysplasia and chronic myeloproliferative disorders. The combination of good morphological preservation and small panels of antibodies (for example, myeloperoxidase, glycophorin C, Factor VIII RAG, and CD34) permits accurate and detailed assessment of bone marrow microarchitecture, which is one of the key features in the diagnosis of myelodyplastic syndrome. ${ }^{18}{ }^{19}$ Myeloblasts can be positively discriminated from early megakaryocytes and erythroid precursors, and their number and distribution assessed. The prognostic importance of determining the numbers of CD34 positive cells in myeloproliferative disorders has been reported. ${ }^{20}{ }^{21}$ The presence of ring sideroblasts can also be readily detected on Perls-stained, MMA-embedded sections.

In conclusion, we suggest that the use of MMA has many advantages over other meth- 
ods of processing bone marrow trephine biopsy specimens, and we would strongly recommend it for routine use.

1 Burkhardt R. Bone marrow and bone tissue. Berlin: Springer Verlag, 1971

2 Moosavi H, Lichtman MA, Donnelly JA, Churukian CJ Plastic-embedding human marrow biopsy specimens. Arch Pathol Lab Med 1981;105:269-73.

3 Beckstead JH. The bone marrow biopsy. Arch Pathol Lab Med 1986;110:175-9

4 Clarke JD. A 24 hour bone marrow biopsy preparation method. Aust f Med Lab Sci 1991;12:138-41.

5 Beckstead JH, Stenberg PE, McEver RP, Shuman MA Bainton DF. Immunohistochemical localization of membrane and $\alpha$-granule proteins in human megakaryocytes: application to plastic-embedded bone marrow biopsy application to plastic-embedded
specimens. Blood 1986;67:285-93.

6 Archimbaud E, Islam A, Preisler HD. Immunoperoxidase detection of myeloid antigens in glycol methacrylateembedded human bone marrow. $\mathcal{f}$ Histochem Cytochem 1987;35:595-9.

7 Islam A, Archimbaud E, Henderson ES, Han T. Glycol methacrylate (GMA) embedding for light microscopy. II. Immunohistochemical analysis of semithin sections of undecalcified marrow cores. F Clin Pathol 1988;41:892-6.

8 Burgio VL, Pignoloni CD. Immunohistology of bone marrow: a modified method of glycol methacrylate embedmarrow: a modifed method of glycol
ding. Histopathology 1991;18:37-43.

9 Vincic L, Colley EC, Beltrano A, Riddell RH. Plastic versus paraffin embedding for histopathology and immunocytochemistry [letter]. Am f Surg Pathol 1990;14:500-1.

10 Schmid C, Isaacson PG. Bone marrow trephine biopsy in lymphoproliferative disease. f Clin Pathol 1992;45:745-50.

11 Pileri S, Poggi S, Baglioni P, Montanari M, Sabatini E, Galieni P, et al. Histology and immunohistology of bone marrow biopsy in multiple myeloma. Eur $\mathcal{f}$ Haematol Suppl 1989;51:52-9.
12 Thiry A, Delvenne P, Fontaine MA, Boniver J. Comparison of bone marrow sections, smears and immunohistological staining for immunoglobulin light chains in the diagnosis of benign and malignant plasma cell proliferations. Histopathology 1993;22:423-8.

13 Reid MM, Wallis JP, McGuckin AG, Pearson AD, Malcolm AJ. Routine histological compared with immunohistological examination of bone marrow trephine biopsy specimens in dissemminated neuroblastoma. $\mathcal{F}$ Clin Pathol 1991;44: $483-6$.

14 Hand NM, Morrell KJ, MacLennan KA. Immunocytochemistry on plastic sections for light microscopy: a novel post-embedding procedure. Proc Roy Microsc Soc 1989;24: A54-5.

15 Hand NM, Morrell KJ. Immunocytochemistry on plastic sections for light microscopy: a new technique. Proc Roy Microsc Soc 1990;25:111.

16 Hand NM, Blythe D, Jackson P. Antigen unmasking using microwave heating on formalin fixed tissue embedded in microwave heating on formalin fixed tissue em

17 Casey TT, Cousar JB, Collins RD. A simplified plastic embedding and immunologic technique for immunophenotypic analysis of human hematopoietic and lymphoid tissues. Am F Pathol 1988;131:183-9.

18 Mangi MH, Mufti GJ. Primary myelodysplastic syndromes: diagnostic and prognostic significance of immunohistochemical assesssement of bone marrow biopsies. Blood 1992;79:192-205.

19 Bartl R, Frisch B, Baumgart R. Morphologic classification of the myelodysplastic syndromes (MDS): combined utilization of bone marrow aspirates and trephine biopsies. Leuk Res 1992;16:15-33.

20 Soligo D, Delia D, Oriani A, Cattoretti G, Orazi A, Bertoll $\mathrm{V}$, et al. Identification of CD $34+$ cells in normal and pathological bone marrow biopsies by QBEND10 monoclonal antibody. Leukemia 1991;5:1026-30.

21 Orazi A, Neiman RS, Cualing H, Heerema NA, John K. CD34 immunostaining of bone marrow biopsy specimen is a reliable way to classify the phases of chronic myeloid leukemia. Am f Clin Pathol 1994;101:426-8. 